will be needed to confirm and investigate the significance of these findings.

Competing interests None declared.

\section{OC-090 DIFFERENT EFFECTS OF FODMAP (FERMENTABLE OLIGO-, DI-, AND MONO-SACCHARIDES, AND POLYOLS) COMPONENTS ON SMALL BOWEL WATER CONTENT: AN MRI STUDY}

doi:10.1136/gutjnl-2012-302514a.90 doi:10.1136/gutjnl-2012-302514a.89

${ }^{1} Y$ Y Lee, ${ }^{2}$ J R H Whiting, ${ }^{1} E$ V Robertson, ${ }^{1} \mathrm{M} H$ Derakhshan, ${ }^{1} \mathrm{~A}$ A Wirz, ${ }^{1} \mathrm{~A}$ Kelman, ${ }^{3} \mathrm{D}$ Morrison, ${ }^{2} \mathrm{P}$ Connolly, ${ }^{1} \mathrm{~K}$ E L McColl. ${ }^{1}$ Institute of Cardiovascular and Medical Sciences, University of Glasgow, Glasgow, UK; ${ }^{2}$ Department of Bioengineering, University of Strathclyde, Glasgow, UK; ${ }^{3}$ Stable Isotope Biochemistry Laboratory, Scottish Universities Environmental Research Centre, Glasgow, UK

Introduction In hiatus hernia there is proximal displacement of the gastro-oesophageal junction (GOJ) relative to the crural diaphragm impairing barrier function. Fluoroscopic studies have indicated proximal migration of the GOJ during TLOSRs but detailed study has been limited by radiation exposure. Using a non-radiological technique we have performed detailed examination of the GOJ during TLOSRs in healthy volunteers.

Methods In twelve subjects, a small magnet $(2 \times 1 \mathrm{~mm})$ was endoscopically clipped to the GOJ and the combined assembly of Hall Effect probe and 36 channel high resolution manometer (Sierra Scientific Inc., USA) was passed nasally. After a 600-calorie test meal (Fortisip, Nurticia, UK), the subjects were studied for $90 \mathrm{~min}$.

Results There was a median of 5 TLOSRs from each of the 12 volunteers. The median (range) amplitude and duration of GOJ migration from start to end of TLOSRs was $4.34 \mathrm{~cm}(1.6-8.8)$ and 23.63s (11.3-41.6) respectively. The proximal migration of GOJ during a TLOSR was characterised by a slow start (phase A) before accelerating (phase B) to peak amplitude. Descent of GOJ started off quickly (phase C) before slowing down (phase D) to baseline. These four different phases of movement are confirmed using mathematical modelling. The polynomial equation (1) and its differential (2) allow the calculation of maximum rate achievable for the four different phases using Microsoft Excel 2010 software:

$$
y=a x^{6}+b x^{5}+c x^{4}+d x^{3}+e x^{2}+f x
$$

$$
d y / d x=6 a x^{5}+5 b x^{4}+4 c x^{3}+3 d x^{2}+2 e x+f
$$

where $y$ is amplitude $(\mathrm{cm}), \mathrm{dy} / \mathrm{dx}$ is the differential of $\mathrm{y}, \mathrm{x}$ is time (s) and a to $f$ are non-linear regression constants. The median (range) velocity of phase $\mathrm{C}$ was $0.92 \mathrm{~cm} / \mathrm{s}(0.33-2.35)$ and this was faster when compared to phase B $0.67 \mathrm{~cm} / \mathrm{s}(0.23-1.73)$, phase D $0.36 \mathrm{~cm} / \mathrm{s}(0.06-0.99)$ and phase A $0.30 \mathrm{~cm} / \mathrm{s}$ $(0.03-2.31)$ respectively; $p<0.0001$. Phase $C$ velocity was strongly correlated with amplitude of GOJ migration with a correlation coefficient of $0.84(p=0.0001)$ but not the other three phases. None of the phases correlated with duration of GOJ migration during TLOSRs.

Conclusion The marked proximal movement of GOJ during TLOSRs represents transient and very severe herniation of the GOJ. Our study also suggests that the initial return of the GOJ to the diaphragmatic hiatus following TLOSRs may be controlled by elastic recoil of the phreno-oesophageal ligament.

Competing interests None declared
${ }^{1} \mathrm{~K}$ Murray, ${ }^{*} \mathrm{~V}$ Wilkinson-Smith, ${ }^{2} \mathrm{C}$ Lam, ${ }^{1} \mathrm{C}$ Hoad, ${ }^{1} \mathrm{E}$ Cox, ${ }^{1} \mathrm{C}$ Costigan, ${ }^{2} \mathrm{~L}$ Marciani, ${ }^{1} \mathrm{P}$ Gowland, ${ }^{2} \mathrm{R}$ Spiller. ${ }^{1}$ Sir Peter Mansfield Magnetic Resonance Centre, University of Nottingham, Nottingham, UK; ${ }^{2}$ NIHR Biomedical Research Unit, Nottingham Digestive Diseases Centre, Nottingham University Hospitals, Nottingham, UK

Introduction A randomised placebo controlled trial (RCT) has shown that FODMAPs exacerbate irritable bowel syndrome (IBS) symptoms in selected patients. FODMAPs are heterogeneous chemically, the major components being fructose and fructans. Both are poorly absorbed in the small bowel but fructose exerts a much greater osmotic effect than fructans which are fructose polymers. Whether this alters the overall impact is unknown. This study aims to test the hypotheses that (a) fructose will increase small bowel water content (SBWC) more than fructans; (b) adding glucose to fructose will reduce its effect, and (c) fructans will exert most of their effect as a result of colonic fermentation.

Methods 4-way, randomised, single blind, crossover study. 16 healthy volunteers underwent abdominal scans in a $1.5 \mathrm{~T} \mathrm{MRI}$ scanner after an overnight fast. They were then fed $500 \mathrm{ml}$ of water containing $40 \mathrm{~g}$ glucose, fructose, fructan or $40 \mathrm{~g}$ glucose plus $40 \mathrm{~g}$ fructose and underwent scanning and breath $\mathrm{H}_{2}$ measurement and completed bowel symptom questionnaires at hourly intervals for $5 \mathrm{~h}$. SBWC was measured from heavily T2 weighted magnetic resonance scans. 1-way analysis of variance was used.

Results (Mean \pm SEM) All drinks were well tolerated with only minor symptoms of gas, bloating, abdominal pain and diarrhoea reported. Relative to glucose, fructose increased SBWC significantly $(184 \pm 35 \mathrm{ml}$ vs $118 \pm 24 \mathrm{ml}$ glucose, $\mathrm{p}=0.001)$, while fructans had little effect ( $\mathrm{SBWC}=119 \pm 23 \mathrm{ml}, \mathrm{p}=0.7$ vs glucose). Adding $40 \mathrm{~g}$ glucose to $40 \mathrm{~g}$ fructose decreased SBWC $(145 \pm 27 \mathrm{ml}$, but the difference was not significant, $\mathrm{p}=0.08$ vs fructose). Breath $\mathrm{H}_{2}$ was initially higher for fructose than the other three solutions. (AUC was $8959 \pm 1880 \mathrm{ppm} . \mathrm{min}$ ) with fructans having the largest AUC at $14987 \pm 2568$ ppm.min). Values returned to baseline for fructose over the $5 \mathrm{~h}$ study, but increased to a maximum for fructan after $300 \mathrm{~min}$. Glucose had no effect on breath $\mathrm{H}_{2}$ concentration, but adding an equivalent amount to fructose significantly reduced breath $\mathrm{H}_{2}$ relative to fructose alone $(\mathrm{p}=0.0006)$.

Conclusion FODMAPs exert a variety of GI responses. Fructose but not fructans increases SBWC relative to glucose. Both fructose and fructans are malabsorbed as confirmed by breath $\mathrm{H}_{2}$ measurements. Fructose malabsorption is significantly reduced by addition of glucose. These different gastrointestinal responses can be readily identified using our non-invasive MRI techniques.

Competing interests None declared.

\section{OC-091 ONDANSETRON SLOWS TRANSIT AND IMPROVES STOOL CONSISTENCY IN PATIENTS WITH DIARRHOEA PREDOMINANT IRRITABLE BOWEL SYNDROME}

doi:10.1136/gutjnl-2012-302514a.91

${ }^{1} \mathrm{~K}$ Garsed, ${ }^{*} \mathrm{M}$ Hastings, 'L Marciani, ${ }^{1} \mathrm{C}$ Lam, ${ }^{1} \mathrm{G}$ Singh, ${ }^{1} \mathrm{M}$ Lingaya, ${ }^{2} \mathrm{P}$ Whorwell, ${ }^{1} \mathrm{R}$ Banwait, ${ }^{1} \mathrm{R}$ Spiller. ${ }^{1}$ Nottingham Digestive Diseases Centre NHIR BRU, University of Nottingham, Nottingham, UK; ${ }^{2}$ Neurogastroenterology and Motility, Wythenshawe Hospital, Manchester, UK

Introduction 5-Hydroxytryptamine three receptor antagonists (5HT3RA) are effective in diarrhoea predominant irritable bowel syndrome (IBS-D), with a number needed to treat (NNT) for 